\title{
ON THE JULIA DIRECTIONS OF THE VALUE DISTRIBUTION OF HOLOMORPHIC CURVES IN $P^{n}(C)$
}

\author{
ZHEN-HAN TU
}

\begin{abstract}
The generalized Picard theorem [4] asserts that any non-constant holomorphic map $f$ of $C$ into $P^{n}(C)$ misses at most $2 n$ hyperplanes in $P^{n}(C)$ in general position. In this paper we shall prove that for a transcendental holomorphic map $f$ of $C$ into $P^{n}(C)$ with an asymptotic value in $P^{n}(C)$, there exists a ray $J(\theta)=\left\{z=r e^{\sqrt{-1} \theta}: 0<r<+\infty\right\}$ such that $f$, in any open sector with vertex $z=0$ containing the ray $J(\theta)$, misses at most $2 n$ hyperplanes in $P^{n}(C)$ in general position.
\end{abstract}

\section{Introduction}

Let $f(z)$ be a non-constant meromorphic function on $C$. We regard $f$ as a holomorphic curve in the Riemann sphere $P(C)$ by identifying $P(C)$ with $C U\{\infty\}$. Picard proved that $f$ misses at most two values in $P(C)$. Using the theory of the normal family, G. Julia [5] proved the following result.

THEOREM A. Let $f(z)$ be a transcendental entire function on $C$. Then there exists a ray $J(\theta)=\left\{z=r e^{\sqrt{-1} \theta}: 0<r<+\infty\right\}$ such that $f$, in any open sector with vertex $z=0$ containing the ray $J(\theta)$, misses at most one value in $C$.

H. Milloux [6] generalized Theorem A to meromorphic functions on $C$ and proved the following result.

THEOREM B. Let $f(z)$ be a transcendental meromorphic function on $C$ with an asymptotic value in $P(C)$. Then there exists a ray $J(\theta)=\left\{z=r e^{\sqrt{-1} \theta}: 0<r<+\infty\right\}$ such that $f$, in any open sector with vertex $z=0$ containing the ray $J(\theta)$, misses at most two values in $P(C)$.

The ray $J(\theta)$ in Theorem $\mathrm{A}$ or Theorem $\mathrm{B}$ is called a Julia direction of $f$. Since a transcendental entire function always has an asymptotic value $\infty$ in $P(C)$, Theorem $\mathrm{B}$ is a generalization of Theorem $\mathrm{A}$. We must note that not every transcendental meromorphic function has a Julia direction. In fact,

Received March 28, 1994; revised June 19, 1995. 
A. Ostrowski [9] ever gave a transcendental meromorphic function on $C$ with no Julia direction. Thus the condition that $f$ has an asymptotic value in Theorem $B$ can not be removed.

Thereafter up to the present, there are many detail researches about the Julia directions (such as [1], [2] and [12]). But all of these studies have been restricted to the entire and meromorphic functions on the complex plane. Its higher dimensional counterpart seems to have been overlooked before.

In this paper, we shall prove the existence of the Julia directions of some holomorphic curves in $P^{n}(C)$. We use here standard notations of the theory of holomorphic curves in $P^{n}(C)$, see, e. g., [8] and [11].

\section{Main result}

A holomorphic curve $f: C \rightarrow P^{n}(C)$ is called an entire holomorphic curve in $P^{n}(C)$. We say that an entire holomorphic curve $f$ has an asymptotic value in $P^{n}(C)$ if there exist a continuous path : $z=z(t)(0 \leqq t<1)$ satisfying $\lim _{t \rightarrow 1} z(t)=\infty$ and a reduced representation $\tilde{f}(z)=\left(f_{0}(z), f_{1}(z), \cdots, f_{n}(z)\right)$ such that $\lim _{t \rightarrow 1} f_{i}(z(t))$ $=a_{\imath}(0 \leqq i \leqq n)$ with the property that $\left(a_{0}, a_{1}, \cdots, a_{n}\right)$ induces a point in $P^{n}(C)$. We must note that if $f$ has another reduced representation $\tilde{f}(z)=\left(g_{0}(z), g_{1}(z)\right.$, $\left.\cdots, g_{n}(z)\right)$ such that $\lim _{t \rightarrow 1} g_{i}(z(t))=b_{i}(0 \leqq i \leqq n)$ exist and $b_{i_{0}} \neq 0$ for some $i_{0}$, then $\left(a_{0}, a_{1}, \cdots, a_{n}\right)$ and $\left(b_{0}, b_{1}, \cdots, b_{n}\right)$ induce the same point in $P^{n}(C)$. If $n=1$, then the definition of the asymptotic value of holomorphic curve in $P^{n}(C)$ is the same as that of meromorphic function on $C$.

The generalized Picard theorem [4] asserts that a non-constant entire holomorphic curve $f$ misses at most $2 n$ hyperplanes in $P^{n}(C)$ in general position (the result was considerably extended in [7] and [10]). In this paper, we shall prove the following main result.

THEOREM 1. Let $f(z)$ be a transcendental entire holomorphic curve with an asymptotic value in $P^{n}(C)$. Then there exists a ray $J(\theta)=\left\{z=r e^{\sqrt{-1} \theta}: 0<r<+\infty\right\}$ such that $f$, in any open sector with vertex $z=0$ containing $J(\theta)$, misses at most $2 n$ hyperplanes in $P^{n}(C)$ in general position.

We call $J(\theta)$ in Theorem 1 to be a Julia direction of holomorphic curve $f$.

\section{Some lemmas}

At first, we give some notations concerning the normal family of holomorphic curves in $P^{n}(C)$ (see [3] for reference).

Let $F$ be a family of holomorphic curves of a domain $U$ in $C$ into $P^{n}(C)$. We call $F$ to be normal in $U$ if any sequence in $F$ contains a subsequence $\left\{f^{(p)}(z)\right\}$ such that each $f^{(p)}(z)$ has a reduced representation

$$
\tilde{f}^{(p)}(z)=\left(f_{0}^{(p)}(z), f_{1}^{(p)}(z), \cdots, f_{n}^{(p)}(z)\right)
$$


in $U$ and $\left\{f_{i}^{(p)}(z)\right\}(0 \leqq i \leqq n)$ converges uniformly on compact subsets of $U$ to a holomorphic function $f_{i}(z)(0 \leqq i \leqq n)$ with $\left(f_{0}(z), f_{1}(z), \cdots, f_{n}(z)\right) \neq 0$ everywhere in $U$. This means that $\left(f_{0}(z), f_{1}(z), \cdots, f_{n}(z)\right)$ induces a holomorphic curve of $U$ into $P^{n}(C)$. We should note the following basic fact (see (3.2) in [3] for reference): If there exists another reduced representation

$$
\tilde{f}^{(p)}(z)=\left(g_{0}^{(p)}(z), g_{1}^{(p)}(z), \cdots, g_{n}^{(p)}(z)\right)
$$

in $U$ such that

$$
\lim _{p \rightarrow \infty} g_{i}^{(p)}(z)=g_{i}(z) \quad(0 \leqq i \leqq n)
$$

exist and $\left(g_{0}(z), g_{1}(z), \cdots, g_{n}(z)\right) \neq 0$ everywhere in $U$, then $\left(f_{0}(z), f_{1}(z), \cdots, f_{n}(z)\right)$ and $\left(g_{0}(z), g_{1}(z), \cdots, g_{n}(z)\right)$ induce the same holomorphic curve of $U$ into $P^{n}(C)$. We call $F$ to be normal at a point $p$ in $U$ if $F$ is normal in some neighborhood of $p$ in $U$.

LEMMA 1. Let $F$ be a family of holomorphic curves of a domain $U$ in $C$ into $P^{n}(C)$. If $F$ is normal at every point in $U$, then $F$ is normal in $U$.

Proof of Lemma 1 follows from the following method: Let $\left\{E_{i}\right\}$ be a sequence of compact subsets of $U$ which satisfies the conditions :

a. If $K$ is a compact subset of $U$, then $K$ is contained in some $E_{\imath}$.

b. $U=\bigcup_{\imath=1}^{\infty} E_{\imath}$.

For any sequence in $F$, by the Heine-Borel finite covering theorem and diagonal method we can take a subsequence which converges uniformly on each $E_{\imath}$ to a holomorphic curve of $U$ into $P^{n}(C)$. We obtain Lemma 1 .

LEMMA 2. Let $F$ be a family of holomorphac curves of $U$ into $P^{n}(C)$. If there exist $2 n+1$ hyperplanes in $P^{n}(C)$ in general position such that every holomorphic curve in $F$ omits these $2 n+1$ hyperplanes, then $F$ is normal in $U$.

Lemma 2 is a well-known basic result. In fact, combining (1.5.8) and (1.8.9) in [8], we immediately have Lemma 2.

LEMMA 3. Let $f(z)$ be an entire holomorphic curve in $P^{n}(C)$ with an asymptotic value $a$ in $P^{n}(C)$. If the sequence $\left\{f\left(2^{n_{k}} z\right)\right\}$ converges uniformly on compact subsets of $1 / 2<|z|<4$ to a holomorphic curve $g(z)$ of $1 / 2<|z|<4$ into $P^{n}(C)$, then $g(z) \equiv a$ in $1 / 2<|z|<4$.

Proof of Lemma 3. Let $z=z(t)(0 \leqq t<1)$ be a continuous path on $C$ satisfying $\lim _{t \rightarrow 1} z(t)=\infty$ and $\lim _{t \rightarrow 1} f(z(t))=a$.

For a given $r(1 / 2<r<4)$, let

$$
S_{r}=\{z \in C:|z|=r\} .
$$

Then there exist $\left\{t_{i}\right\} \quad\left(0<t_{1}<t_{2}<\cdots<1\right.$ and $\left.\lim _{k \rightarrow \infty} t_{k}=1\right)$ and $\left\{z_{k}\right\} \subset S_{r}$ such that 
$z\left(t_{k}\right)=2^{n_{k}} z_{k}(k=1,2, \cdots)$. Thus we have

$$
\lim _{k \rightarrow \infty} f\left(2^{n_{k}} z_{k}\right)=\lim _{k \rightarrow \infty} f\left(z\left(t_{k}\right)\right)=a .
$$

without loss of generality, we assume $\lim _{k \rightarrow \infty} z_{k}=z_{0}\left(\in S_{r}\right)$. Since $\left\{f\left(2^{n_{k}} z\right)\right\}$ converges uniformly on compact subsets of $1 / 2<|z|<4$ to $g(z)$, we have

$$
g\left(z_{0}\right)=\lim _{k \rightarrow \infty} f\left(2^{n_{k}} z_{k}\right)=a .
$$

Since $r$ is arbitrary in $1 / 2<r<4$, by the uniqueness theorem of meromorphic functions in one variable we get $g(z) \equiv a$ in $1 / 2<|z|<4$. Lemma 3 is proved.

LEMMA 4. Let $f(z)$ be a transcendental entire holomorphic curve in $P^{n}(C)$ with an asymptotic value $a$ in $P^{n}(C)$. Then the sequence of holomorphic curves $\left\{f\left(2^{n} z\right)\right\}$ of the domain $1 / 2<|z|<4$ into $P^{n}(C)$ is not normal in $1 / 2<|z|<4$.

Proof of Lemma 4. Let $\tilde{a}=\left(a_{0}, a_{1}, \cdots, a_{n}\right)$ and $\tilde{f}(z)=\left(f_{0}(z), f_{1}(z), \cdots, f_{n}(z)\right)$ be reduced representations of $a$ and $f(z)$ in $C$ respectively. Then we have some $a_{\imath_{0}} \neq 0$ and then $f_{\imath_{0}}(z) \not \equiv 0$ in $C$. Since $f(z)$ is a transcendental entire holomorphic curve, there exists some $f_{\jmath_{0}}(z)\left(0 \leqq j_{0} \leqq n\right)$ such that $f_{\jmath_{0}}(z) / f_{\imath_{0}}(z)$ is a transcendental meromorphic function on $C$.

I. We assume that $f_{\jmath_{0}}(z) / f_{\imath_{0}}(z)$ has no pole point in $R \leqq|z|<\infty$ for some $R>0$. If $\left\{f\left(2^{n} z\right)\right\}$ is normal in $1 / 2<|z|<4$, then there exists a subsequence $\left\{f\left(2^{n_{k}} z\right)\right\}$ which converges uniformly on compact subsets of $1 / 2<|z|<4$ to a holomorphic curve of $1 / 2<|z|<4$ into $P^{n}(C)$. By Lemma 3 we have

$$
\lim _{k \rightarrow \infty} f\left(2^{n_{k}} z\right) \equiv a
$$

in $1 / 2<|z|<4$. Then we have

$$
\lim _{k \rightarrow \infty} f_{\jmath_{0}}\left(2^{n_{k}} z\right) / f_{\imath_{0}}\left(2^{n_{k}} z\right) \equiv a_{\jmath_{0}} / a_{\imath_{0}}
$$

in $1 / 2<|z|<4$. Thus we can easily get that the holomorphic function $f_{0_{0}}(z) / f_{\imath_{0}}(z)$ in $R \leqq|z|<\infty$ is uniformly bounded. Since $f_{\jmath_{0}}(z) / f_{\imath_{0}}(z)$ on $C$ is a transcendental meromorphic function, this is a contradiction.

II. We assume that there exists $\left\{z_{k}\right\} \subset C$ such that

$$
\lim _{k \rightarrow \infty} z_{k}=\infty \text { and } f_{\jmath_{0}}\left(z_{k}\right) / f_{\imath_{0}}\left(z_{k}\right)=\infty .
$$

Then there exists a subsequence

$$
\left\{f_{\jmath_{0}}\left(2^{n_{k}} z\right) / f_{\imath_{0}}\left(2^{n_{k}} z\right)\right\}
$$

defined in $1 / 2<|z|<4$ such that every $f_{\jmath_{0}}\left(2^{n_{k}} z\right) / f_{\imath_{0}}\left(2^{n_{k}} z\right)(k=1,2, \cdots)$ has at least one pole point $p_{k}$ in $1 \leqq|z| \leqq 2$.

If $\left\{f\left(2^{n} z\right)\right\}$ is normal in $1 / 2<|z|<4$, without loss of generality we assume that $\left\{f\left(2^{n_{k}} z\right)\right\}$ converges uniformly on compact subsets of $1 / 2<|z|<4$ to a 
holomorphic curve of $1 / 2<|z|<4$ into $P^{n}(C)$ and $\lim _{k \rightarrow \infty} p_{k}=z_{0}\left(1 \leqq\left|z_{0}\right| \leqq 2\right)$. By Lemma 3 we have $\lim _{k \rightarrow \infty} f\left(2^{n_{k}} z\right) \equiv a$ in $1 / 2<|z|<4$. Then $\left\{f\left(2^{n_{k}} z\right)\right\}$ converges uniformly on compact subsets of $1 / 2<|z|<4$ to the point a in $P^{n}(C)$. So we have

$$
\lim _{k \rightarrow \infty} f\left(2^{n_{k}} p_{k}\right)=\lim _{k \rightarrow \infty} f\left(2^{n} z_{0}\right)=a .
$$

But on the other hand we have

$$
f_{\jmath_{0}}\left(2^{n_{k}} p_{k}\right) / f_{\imath_{0}}\left(2^{n_{k}} p_{k}\right)=\infty \quad(k=1,2, \cdots)
$$

and $a_{\jmath_{0}} / a_{\imath_{0}}$ is finite. This is a contradiction. Hence we get Lemma 4.

\section{Proof of Theorem 1}

By Lemma 4, we have that $\left\{f\left(2^{n} z\right)\right\}$ is not normal in $1 / 2<|z|<4$. By Lemma 1 there exists at least a point $z_{0}$ in $1 / 2<|z|<4$ such that $\left\{f\left(2^{n} z\right)\right\}$ is not normal at $z_{0}$.

Considering any a small disc $D_{r}\left(z_{0}\right)=\left\{z \in C:\left|z-z_{0}\right|<r\right\}$ in $1 / 2<|z|<4$, by Lemma 2 we have that there exist at most $2 n$ hyperplanes in $P^{n}(C)$ in general position such that every holomorphic curve $f\left(2^{n} z\right)$ defined in $D_{r}\left(z_{0}\right)$ misses these $2 n$ hyperplanes.

Since

$$
\begin{aligned}
& \left\{f\left(2^{n} z\right):\left|z-z_{0}\right|<r\right\} \\
= & \left\{f(z):\left|z-2^{n} z_{0}\right|<2^{n} r\right\} \\
\subset & \left\{f(z):\left|\arg z-\arg z_{0}\right|<\arcsin \left(r /\left|z_{0}\right|\right)\right\},
\end{aligned}
$$

we have that $f(z)$, in any open sector with vertex $z=0$ containing $J\left(\theta_{0}\right)$ : $=\left\{z=r e^{\sqrt{-1}} \theta_{0}: 0<r<+\infty\right\}$ where $\theta_{0}=\arg z_{0}$, misses at most $2 n$ hyperplanes in $P^{n}(C)$ in general position.

We obtain Theorem 1.

Remark. Let $f(z)$ be an entire holomorphic curve in $P^{n}(C)$ with a finite positive order. If the order of $N_{f}(H, r)$ where $H$ is a hyperplane in $P^{n}(C)$ is less than the order of $f(z)$, then $H$ is called a Borel exceptional hyperplane. By the main result in [7] or [10] we have that $f(z)$ has at most $2 n$ Borel exceptional hyperplanes in $P^{n}(C)$ in general position. We call $J\left(\theta_{0}\right)$ to be a Borel direction of $f(z)$ if $f(z)$, in any open sector with vertex $z=0$ containing the ray $J\left(\theta_{0}\right)$, has at most $2 n$ Borel exceptional hyperplanes in $P^{n}(C)$ in general position. A more interesting problem is to prove the existence of the Borel directions of some holomorphic curves in $P^{n}(C)$.

Acknowledgement. I thank the referee for his useful comments on this paper. 


\section{REFERENCES}

[1] J. Anderson and J. Clunie, Entire functions of finite order and line of Julia, Math. Z., 112 (1969), 59-73.

[2] D. Drasin and A. Weitsman, On the Julie directions and Borel directions of entire functions, Proc. London Math. Soc., 32 (1976), 199-212.

[3] H. Fujimoto, On families of meromorphic maps into the complex projective space, Nagoya Math. J., 54 (1974), 21-51.

[4] M. GReEN, Holomorphic maps into complex projective space omitting hyperplanes, Trans. Amer. Math. Soc., 169 (1972), 89-103.

[5] G. Julia, Sur quelques propriétés nouvelles des fonctions entières ou méromorphes, Ann. École Norm. Sup., 36 (1916), 93-125. Ibid., 37 (1920), 165-218.

[6] H. Milloux, Le théorème de Picard, suites de fonctions holomorphes, fonctions holomorphes et fonctions entières, J. Math. Pures Appl., 3 (1924), 345-401.

[7] E. NochkA, On the theory of meromorphic functions, Soviet Math. Dokl., 27 (1983), 377-381.

[8] J. Noguchi and T. OchiaI, Geometric Function Theory in Several Complex Variables, Transl. Math. Monographs, 80, Amer. Math. Soc., Providence, RI, 1990.

[9] A. OstrowsKI, Über Folgen analytischer Functionen und einige Verschärfungen des Picardschen Satzes, Math. Z., 24 (1926), 215-258.

[10] M. Ru AND W. Stoll, The Cartan conjecture for moving targets, Several Complex Variables and Complex Geometry, Proc. Sympos. Pure Math., 52, Part 2, 1991, 477-508.

[11] H. Wu, The Equidistribution Theory of Holomorphic Curves, Ann. of Math. Studies, 64, Princeton Univ. Press, Princeton, 1970.

[12] H. Yoshida, Julia directions of entire functions of smooth growth, Nagoya Math. J., 87 (1982), 41-57.

Department of Mathematics

HuAzhong University of SCIENCE AND TEChNology

WuHAN, Hubei 430074

People's Republic of China 